\title{
Contents, Vol. 44, 1920
}

lnhalts - Verzeichnis.

S $\theta i t \beta$

Or $1 / 8$ inalarbeiten.

Bachstez, E., u. H. Purtseher, Übe.i die Häufung retrobulbärerNeuritiden einschließlich toxischer Amblyopien gegen-über der Zeit vor dem Kriege 38

Birch-Hirschfeld, Gustav, Zur Behandlung entzündlicherHornhauterkrankungen, besonders des Ulcus serpens, mit ultraviolettem Licht 1

Comberg, W., Über Badkonjunktivitis (Hierzu Tafel I) . . 13

Esser, Albert, Z,wc Genese der akkomodativen Mikropsie

und Makropsie 132

Feilchenfeld, W., Einfluß des Krieges auf die Akkommo-

dationsfähigkeit $\quad 140$

Fertig, A., Zur Frage der traumatischen Keratitis parenchy-

matosa und ihrer Begutaehtung in der Unfallpraxis . 166

Fischoeder, E., Über Schädigungen am vorderen Augen-abschnitt durch Röntgenstrahlen an einem wegen Ader-hautsarkoms bestrahlten Bulbus 160

Friede, Iîeinh., Über einen Fall von Pityriasis lichenoides

chronica der Lider und der Konjunktiva 253

(riúst, Gustav, Ein Beitrag zur Klinik der Haabschen Bän-

dertrübung 242

Holler, G., Über Proteinkörpertherapie 145

Junius, Paul, Kann Grippe rerlektorische Pupillenstarre be-m dingen? 44

7-- Neuere Ansichten über den Herpes zoster (Herpes communis, ophthalmicus, traumatieus, dem Herpes verwandte Erkrankungen der Hornhaut) ..... 74

- - - Die Probleme der Vererbung und der Erwerbung

der Kurzsichtigkeit 262

'Kraupa, Ernst, Pigmentierung der Hornhauthinterfläche bei

““ „Dystrophia epithelialis” (Fuchs) 247

- - Über Vergiftungserscheinungen in Form epileptischerKrämpfe bei Anwendung von

Novokainlösungen zurLeîtungsunempfindliehkeit der Augenhöhle 250

Marburg, Otto, Retrobulbäre Neuritis optica und multiple

Sklerose 125

Schnyder, Walter F., Über familiäres Vorkommen resp. die

Vererbung von Erkrankungen der Tränenwege . . . 257

$-\mathrm{IV}$ -

Seite

Vogt, A., Die Diagnose partieller und totaler Vorderkammer-

aufhebung mittels Spaltlampenmikroskop 237

de Weldige-Cremer, Über einen Fall von Lymphangioma 
cysticum der Orbita (Hierzu Taf. III.).65

Wick, Willy, Kolobom am Sehnerveneintritt (HierzuTafel II) 51

Wirths, M., Beiderseitige Lidgeschwulst, kombiniert mit Ge-

schwulstbildung der Oberlippe 17ü

Zade, M., Über intermittierenden Exophthalmus .... 153

liericht über die deutsche ophthnlmologische $L \cdot$ iteratur.

Die angeborenen Anomalien und Mißbildungen des Auges.

Von Prof. Dr. E. See/elder in Innsbruck 90

liericht über die ausländische ophlhalmologisclie Literatur.

Holländische ophthalmologische Literatur. 1918. 2. Hälfte.Von Dr. G. J. Schoute in Amsterdam und Prof. Dr.W. Koster Gzn im Haag96

Gesellschaftsberichte.

Bericht über die XLII. Zusammenkunft der DeutschenOphthalmologischen Gesellschaft in

Heidelberg am5. 6. und 7. Aug. 1920. Nach Autoreferaten erstattetvon Dr. Junius 303

Verein der Augenärzte von Ost- und Westpreußen. Sitzung

am 28. Febr. 1920 in Königsberg i. Pr

329

Ophthalmologische Gesellschaft in Wien. Sitzung vom

17. Nov. und 15. Dez. 1919101

-- - Sitzungen vom 19. L, 16. II., 1. III., 15. III. und

19. IV. $20 \quad 178$

- - Sitzung vom 17. Mai $1920 \quad 335$

ünfall- und Vcrsicherimgskuude 113,336

Diagnostische Notizen 231

Therapeutische Umschau 117, 222, 339

Literaturverzeichiiis $118,233,345$

lierichtigung 348

Offene Korrespondenz

Tagesnachrichten und Personalien 123, 348

Hermann Ulbrich $\dagger \quad 344$

S. Sachregister fur Band XLIП und XLIV 349

352

Namenregister fur Band XLIII und XLIV .... 351

355 